\title{
An Applied Method of Gaussian Noise by Fuzzy Filter
}

\author{
Anita Pati Mishra,Sangita Gupta, Vini Malik \\ Department of Mathematics,Department of Mathematics,Department of Electronics \&Telecommunication \\ CET-IILM-AHL Greater Noida
}

\begin{abstract}
The world has witnessed a major change in the way computing systems are used, especially to process images. DIP is a prominent area of research in the field of information technology that helps in processing digital images captured through various modalities using computing algorithms. Image processing has revolutionized greately thanks to its versatile applications and scalability. It not only helps to enhance images for better human interpretation but also allows processing images for storage and retrieval to be used for different representation and analysis. DIP techniques are generally versatile, reliable and accurate and additional benefit of being easier to implement than analog forms. The application of DIP pervades through various domains such as biology, Robotics. DIP uses size, color, shape and texture of objects under consideration for knowledge discovery. Respective domain knowledge is an important aspect when studying DIP. Image processing methods are numerous and varied and are used as per requirement in every field it is applied in. Techniques that were computationally prohibitive are now becoming reasonable within a DIP environment. Detection of edges in an image is very important step towards understanding image features. Edges are often considered as object boundaries at image locations. So edge detection is extensively used in image segmentation when images are divided into areas corresponding to different objects. This can be specifically used for enhancing tumor area in mammographic images. Different methods are available like laplacian \& Gaussian edge operator. Breast boundaries, pectoral region and tumor location can be seen clearly by this method. Image noise suppression is highly demanding approach in DIP. Impulse noise is one which creates problem in acquisition transmission and processing of images. When image is restored filter act on 2 major roles .(1) Classification or detection and (2) Reconstruction of images which is filtering. Reconstruction involves replacing of corrupted pixels by certain approximation technique
\end{abstract}

Keywords: Additive noise, moving window, fuzzy inference rule, peak signal to noise ratio

\section{Introduction}

The application of DIP pervades through various domains such as medicine and biology, multimedia, robotics,photoand video, security and surveillance,remote sensing, biometrics etc. Now I will recollect that DIP is used in the above mentioned areas for the following functions.

1. To analyze the gene expressions of the DNA microarray images.

2. Powerful algorithms are developed for the analysis of multimedia data from digital evidences to take decisions in information forensics.

3. DIP plays an important role in the medical field.The prominent areas where DIP is extensively used are in the diagnosis and detection of various diseases and problems using digital images obtained through Xrays,CT scanMRI, PET scan etc.

4. Researchers of archaeology are approaching computer scientists to help them develop their findings through excavations using archived photographs.

5. Remote sensing is a major area where image processing plays a crucial role in determining the natural calamities as well as day to day weather and climatic conditions.

A number of researches have been conducted and applications are developed based on these research reports, to combine DIP with other fields of computer science such as genetic algorithms neural networks and fuzzy systems etc. it is important for students and professionals in the field of DIP to conduct further research that will help create an adaptable and resilient technology paving the way for a new easy and cost effective method of decision making based on digital images.In this paper a new fuzzy mechanism is developed for noise reduction of images corrupted by additive noise as well as protecting the edges. The proposed mechanism consists of 3 stages.(1). Define fuzzy sets in the input space to compute fuzzy derivative.(2).To construct if then else rule to perform fuzzy smoothing according to the contribution of surrounding pixel values (3). Define fuzzy sets in the outer space to get maximum noise free and edged image.

Experimental results are obtained to show the feasibility of the proposed filter for two dimensional images. 


\section{Conceptual Implementation :}

Noise Model : There are 2 randomly corrupted pixels. The extreme value $0 \& 255(8$ bit monochrome image with same derivation) probability.For each pixel in the image in S.I space with intensity value(I,J) j,If the image is noisy pixels $X^{\prime}$.

\section{Gaussian Noise function}

\section{$P=$ density of Image data}

$\mathrm{P}(\mathrm{z})=\frac{1}{2 \pi \sigma} e^{-(z-\mu)^{2} / 2 \sigma \wedge 2}$

$\mathrm{P}=$ density of Image data, $\mathrm{z}=$ gray level, $\sigma=$ Standard deviation,$\sigma^{\wedge} 2=$ Variance.

Detection of edges in an image is very important step towards understanding image features. Edges are often considered as object boundaries at image locations. So edge detection is extensively used in image segmentation when images are divided into areas corresponding to different objects. This can be specifically used for enhancing tumor area in mammographic images. Different methods are available like laplacian \& Gaussian edge operator. Breast boundaries, pectoral region and tumor location can be seen clearly by this method.

Image noise suppression is highly demanding approach in DIP. Impulse noise is one which creates problem in acquisition,transmission and processing of images. When image is restored filter act on 2 major roles .(1) Classification or detection and (2) Reconstruction of images which is filtering. Reconstruction involves replacing of corrupted pixels by certain approximation technique.

The various image processing techniques employed such as linear scaling,median filteres, non linear filters, optical methods, Gabor's filter have not been able to handle the disturbances occurring in processing an image .Hence Fuzzy techniques, fuzzy mechanisms, fuzze enhancement classifier functions, fuzzy inference rules etc. used in processing an image, have evolved as the most efficient solution for this problem. These techniques with fuzzy sets give much-improved image compared to the others. Fuzzy techniques help us to cope up with the day-to-day problems arising in the field of image .An image is defined as two dimensional function of $f(x, y)$, where $x, y$ are spatial co-ordinates. Amplitude of $f(x, y)$ at any pair co-ordinates of $x, y$ is called the intensity or gray level of image at that point.

The useful methods in determining an image type is to consider three types of computerized processors-low, mid and high level.

Low-level processors involve primitive operations such as image processing to reduce noise, contrast enhancement and sharpening. They are characterized by the fact that both its input and outputs are images. Mid level processing of images involves tasks such as segmentation-(partitioning image into regions or objects).It characterized by the fact that its inputs are images but its output are attributes extracted from those images.High level involves "making sense" of an ensemble of recognized object as an image analysis and performs cognitive functions normally associated with vision.

\section{TYPICAL PROBLEMS:}

Some of the most commonly noticed problem while working with images are:

- Geometric transformations such as enlargement, reduction, and rotation

- Color corrections such as brightness and contrast adjustments, quantization, or conversion to a different color space

- Registration (or alignment) of two or more images

- Combination of two or more images, e.g. into an average, blend, difference, or image composite

- Interpolation, demosaicking, and recovery of a full image from a RAW image format like a Bayer filter pattern

- Segmentation of the image into regions

- Image editing and digital retouching

Besides static two-dimensional images, the field also covers the processing of time-varying signals such as video and the output of tomographic equipment. Techniques such as morphological image processing are specific to binary or gray scale images.

IF the pixel is white AND its neighborhood is also white AND homogeneous

THEN it belongs to the background.

Fuzzy Integrals functions can be used in different forms:

Segmentation by weighing the features (fuzzy measures represent the importance of particular features)

Fusion of the results of different segmentation algorithms (optimal use of individual advantages)

Segmentation by fusion of different sensors (e.g. multispectral images, fuzzy measures represent the relevance/importance of each sensor) 
Measures of Fuzziness and image information Measures of fuzziness (e.g. fuzzy entropy) and image information (e.g. fuzzy divergence) can be also used in segmentation and threshold tasks..

Fuzzy Geometry Fuzzy geometrical measures such as fuzzy compactness and index of area coverage can be used to measure the geometrical fuzziness of different regions of an image. The optimization of these measure (e.g. minimization of fuzzy compactness regarding to the cross-over point of membership function) can be applied to make fuzzy and/or crisp pixel classification .

What is Fuzzy Inference Rule:-

It is a process of formulating a mapping from given input data to output data using fuzzy logic.

The function provides a basis from which decisions can be made.

The components of FIR describes as

(i) Membership functions

(ii) Logical operation

(iii) If then rule.

All rules are evaluated as fuzzy reasoning methods.The results of rules are combined and defuzzification process strats.

The algorithm of FIR as follows.

(1) Fuzzification of input variable

(2) Application of fuzzy operator(AND/OR) in the antecedent

(3) Implimentation of antecedent to result

(4) Aggregation of results across the rule

(5) Defuzzification

So FIR displays all parts of FIP from fuzzification through defuzzification.
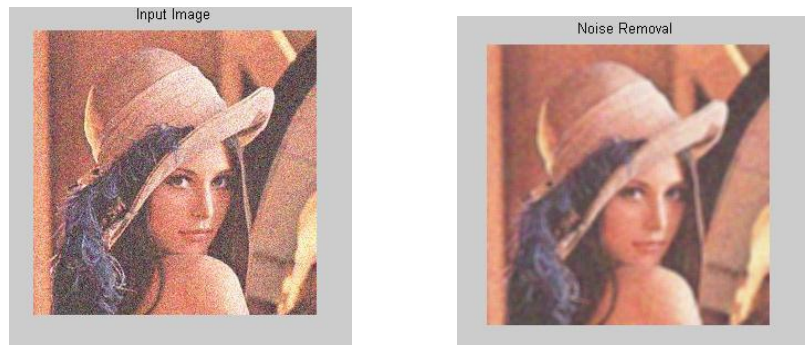

In this paper a novel approach for de noising images corrupted by random valued impulses has been proposed. Noise suppression is done in two steps. The detection of noisy pixels is done using all neighbor hood directional weighted pixels (ANDWP) in the $5 \mathrm{x} 5$ window. The filtering scheme is based on minimum variance of the nine directional pixels.

\section{Vision system:}

\section{Section - II}

Image acquisition is an important process for quality control because it provides the input data for the whole process. Due to rapid progress in the modern manufacturing technology reliable vision systems provide high speed flexible designing systems are a must. The proposed vision systems is based on a matrix a CCD camera as an image acquisition system. The captured colored image is converted to gray image and then algorithms are applied on that image.

Before a vision system can be applied to an image data in order to filter some specific piece of image data, it is usually necessary to process the data in order to assure that it satisfies certain conditions implied by the method.

1, Re sampling in order to assure that image coordinate system is correct. 2.Noise function can be created such as random noise function or gaussian noise function or sensor noise which is to be added as to disturb the captured image data.3. Contrast enhancement techniques to assure the relevant information.4. Detection of noise and then removal of higher intensity value from the pixel data.5. Protecting the whole image to get the most approximate appearance of the original image. 
method. All the gradient based algorithms have operators that calculate the value of the slope in the direction which are orthogonal to each other commonly vertical and horizontal. Then the different components of the slopes are combined to give the total value of the edge strength.

The system implementation was carried out considering the both input and output image obtained after defuzzfication. Some of the methods are chosen as the defuzzification procedure which means that the output fuzzy sets are obtained by applying each inference rule to the input data. The output fuzzy sets are obtained by applying each inference rule to the input data were joined through the addition function, the output of the system was then computed as the centroid of the resulting membership function is most important entity to detect presence of noise in the proposed image.

1. Add the salt and pepper noise or Gaussian noise to the gray level image which can be applied by MATLAB standard functions.

2. Implementing fuzzy membership function to the noisy image which is called fuzzification..

3. designing the fuzzy mechanism rules

4. At the same time values are checked to protect the edges of the testing image.

5. Implementing the output membership functions called defuzzification.

The resulting matrix represents the output values of the proposed fuzzy mechanisms of the input gray level pixels.The resulting image is the filtered image of noisy data with extracted edges of the features of the testing image. where $i, j$ denotes edge detector values (row-column value of testing window).

$\mu(\mathrm{x}, \mathrm{y})$ denotes the membership function at point $(\mathrm{x}, \mathrm{y})$. The derivative value is denoted by $\Delta(\mathrm{x}, \mathrm{y})$. The Gaussian noise removal using fuzzy derivative is denoted as shown below.

$\Delta(\mathrm{x}, \mathrm{y})=\mathrm{f}(\mathrm{x}, \mathrm{y}+1)-\mathrm{f}(\mathrm{x}, \mathrm{y})$

6.The idea behind the Gaussian function is that if no edge is assumed to be present in a certain direction, the derivative value of the function is used. For importance of filtering, positive and negative values are distinguished using if $\Delta(\mathrm{x}, \mathrm{y})$ is positive then mark that "presence of noise" else if $\Delta(\mathrm{x}, \mathrm{y})$ is negative which indicates "absence of noise" To find out the performance of the proposed FIR simulations are carried out in an extensive manner under different noisy conditions in MATLAB using the standard color image of lena \& clown. The performance is evaluated on the basis of PSNR and MSE.

FIR function $=(\mathrm{x} 11 * \mathrm{x} 22 * \mathrm{x} 33)+(\mathrm{x} 31 * \mathrm{x} 22 * \mathrm{x} 13) / 2$;

\section{Experiment \& Simulations (Gaussian noise detection and filtering) -}

The proposed filter is applied to $(256,256)$ clown image and $(120,120)$ Lena Image after adding salt and pepper noise and Gaussian noise at different levels. These noises can be due to fluctuations of light or photoelectric effect of sensors. All images have noise it may be due to climatic effect or any kind of troubles. We can chow the practical approach of our proposed filter which includes original gear image at left side and then noisy image at right hand side and the filtered one. To verify the result of the proposed filter (FIR)we can add Gaussian noise to the image data and the verify the proposed algorithm. The proposed filter removed maximum noise and at the same time it protected the edges to maintain its originality. Finally we ensure the robustness of the proposed filter another image can be tested after adding random salt and pepper noise and then Gaussian noise.

Performance of the proposed Gaussian Noise removal using new Fuzzy inference rule is measured by following parameters.

(i) PSNR

(ii) MSE

Measures of PSNR

Graph:

\begin{tabular}{llll}
\multicolumn{1}{c}{ PSNR } & & Median & \\
proposed EFC & Entrolpy filter & filter & DD filter \\
13.11 & 12.5 & 12 & 10.5 \\
19.25 & 18.25 & 17 & 15 \\
24.3 & 19 & 18.5 & 16.75 \\
30 & 22 & 20 & 19
\end{tabular}




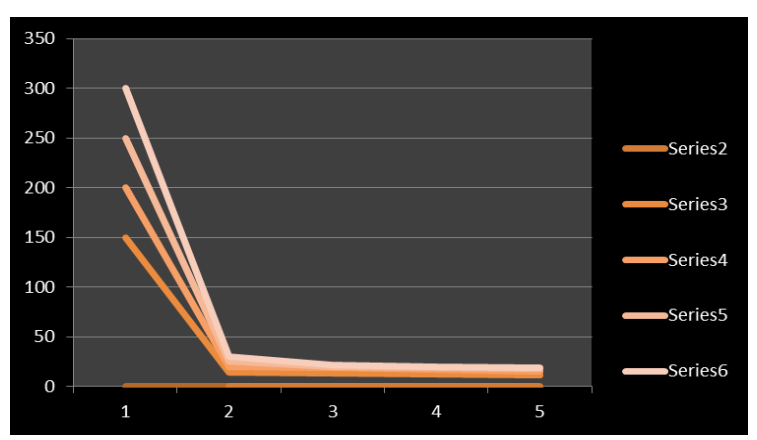

The above graphs show the MSE obtained in lena image. This is a comparison analysis using Double derivative filter, fuzzy logic filter and fuzzy inference rule-filter with error ratio less in our new method. We observe that degration is quite less as compared to other filters.

\section{Conclusion}

The role of fuzzy logic in representing and managing the uncertainties in these tasks was explained. A new approach named as Fuzzy Inference rule has been applied specially for removal of Gaussian noise from 2D Images. We analyze this paper proposed not only a new fuzzy filter but also edge detector method. The main advantage is that it distinguishes between local variations due to noise and image structures using this fuzzy rule. It is relatively simple and less computations for implementation. Experimental results show the feasibility of the new filter and a simple detection and filtration mechanism so finally this fuzzy mechanism enables fast hardware implementation.our next research will certainly concentrate on more efficient fuzzy mechanisms to detect more complex disturbance and minimize it as far as possible.

\section{References}

[1]. E. Kerre and M. Nachtegael, ed, Fuzzy techniques in image processing, New York: Springer,verlog, vol-52,2000.

[2]. H.K.Kwan and Y. Cai,'”Fuzzy filtering for image filtering,' presented in proc.MWSCAS,pp 672-675,2002

[3]. Gonzalez and Woods,Digital Image Processing, Third Edition,Pearson Education 2008.

[4]. M. S. Alani, Digital Image Processing using Matlab university Bookshop,sharqa,USA 2008

[5]. S.Mohapatra, P. K. Sa and B Majhi,Impulsive noise Removal Image Enhancement Tech, $6^{\text {th }}$ WSE As (ICCSE,control \& signal processing (CSE CS 2007) 2007,Cairo,Egypt.

[6]. Karnik N.N and Mendel, J.M 4 centroid of a type-2 fuzzy set, “ International journal on information sciences".

[7]. www.mathworks.in

[8]. A.K. Jain, fundamentals of digital Image Processing, Engle wood cliff, N.J.Prentice Hall 1989. 